\title{
Dynamics of uncaught foil-stripped electrons in the Oak Ridge Spallation Neutron Source accumulator ring
}

\author{
S. Cousineau, J. A. Holmes, M. A. Plum, and W. Lu \\ Oak Ridge National Laboratory, Oak Ridge, Tennessee 37830, USA
}

(Received 17 January 2011; published 24 June 2011)

\begin{abstract}
The Spallation Neutron Source accelerator utilizes $\mathrm{H}^{-}$charge exchange injection to merge a $1 \mathrm{GeV}$, $1 \mathrm{MW}$ beam from a linac into an accumulator ring. The design calls for the $545 \mathrm{keV}$ foil-stripped electrons to be absorbed by an electron catcher located below the foil. However, multiple observations of hardware damage caused by stripped electrons indicate that at least a fraction of the electrons are reflected back into the vacuum chamber. This paper presents the results of a 3D computational model employed to simulate the dynamics of foil-stripped electrons, including a surface interaction model for the catcher. Simulation results are compared with experimental observations, and the implications for high power beam operations are discussed.
\end{abstract}

DOI: 10.1103/PhysRevSTAB.14.064001

PACS numbers: 41.85.Ar, 41.75.- $\mathrm{i}$

\section{INTRODUCTION}

Many high power hadron accelerators utilize $\mathrm{H}^{-}$charge exchange injection to transfer beams from one section of the accelerator to the next, often from a linac into a ring [1]. This method of injection is ideal for high intensity beam accumulation because it minimizes emittance blowup. The process of foil stripping at high beam power poses a number of challenges, including foil heating and foil stability, beam scattering, transport of waste beams, and disposal of stripped electrons. Recently, a great deal of attention has been paid to the development of novel stripping techniques $[2,3]$, and to the fabrication of more resilient foils $[4,5]$. Less effort has been dedicated to understanding the dynamics of the foil-stripped electrons, especially in operational machines. Although the electrons constitute only a fraction of the beam power contained in the parent ion beam, for high power beams this fraction is sufficient to cause damage to machine hardware, unless properly handled.

The Spallation Neutron Source (SNS) is a $1.4 \mathrm{MW}$ accelerator used for neutron production. The accelerator is composed of a $1 \mathrm{GeV} \mathrm{H}^{-}$linac, a proton accumulator ring designed to accumulate up to $1.5 \times 10^{14} \mathrm{ppp}$, and associated transport lines. Commissioning of the SNS was completed in 2006, and the accelerator is currently running with $1 \mathrm{MW}$ of beam power. The SNS is first in a new generation of proton drivers that will deliver megawatts of beam power for use in various secondary systems. Thus, the SNS provides an important point of reference for future high power hadron machines.

Published by the American Physical Society under the terms of the Creative Commons Attribution 3.0 License. Further distribution of this work must maintain attribution to the author(s) and the published article's title, journal citation, and DOI.
The SNS utilizes charge exchange injection to merge the $\mathrm{H}^{-}$beam from the linac with the circulating proton beam in the accumulator ring. An electron catcher intended to absorb the electrons is installed in the region below the foil. The stripped electron beam represents $\sim 1 \mathrm{~kW}$ of beam power, enough to damage the machine if not properly handled. Unfortunately, the installed position of the catcher differs from the design specification by several millimeters. This, together with the fact that the injected beam spot has been relocated twice since the start of operations, compromises the efficiency of the catcher system. Recent experimental observations, such as black marks on the top surface of the catcher and damage to the injection foil assembly, have lead to the hypothesis that a significant portion of stripped electrons scatter back into the vacuum chamber and intercept local hardware.

This paper presents the results of a study to investigate this hypothesis. Using 3D computational methods and a surface reflection model based on MCNPX [6] calculations, the trajectories of the SNS foil-stripped electrons have been modeled. This work considers only the electrons that constitute catcher inefficiency, i.e., those that are not captured by the electron catcher in the intended fashion and consequently pose a threat to the machine. The exact level of catcher inefficiency in the real machine is unknown and has likely varied with the injected beam position. Two specific case scenarios, one for the current SNS injection configuration, and one for a previous configuration, have been simulated and compared with experimental findings.

The paper is organized as follows. Section II provides a review of the relevant injection system parameters. Section III presents the experimental observations that motivated the study. Section IV provides a conceptual framework for the electron motion. Section V describes the full 3D computational model. Section VI presents the results of simulations and comparisons with experimental observations, as well as an estimate of errors. Section VII 
discusses implications for the SNS and other future high power proton drivers.

\section{THE SNS RING INJECTION AND ELECTRON CATCHER SYSTEM}

Injection into the SNS accumulator ring is accomplished by passing the $\mathrm{H}^{-}$beam from the linear accelerator through a $0.35 \mathrm{mg} / \mathrm{cm}^{2}$ thick carbon graphite or diamond stripping foil [4]. The foil is located approximately $79 \%$ of the way through the second of four injection chicane dipole magnets, and is tilted $30^{\circ}$ from the transverse plane about the vertical axis. The four chicane magnets were designed to meet a list of field requirements aimed at minimizing injection beam loss [7]. The second chicane magnet, which contains the foil, was designed to collect the stripped electrons. The main field component of the magnet is $0.25 \mathrm{~T}$. In order to produce pitched field lines that guide the electrons downward to the bottom of the aperture, the bottom pole of this magnet was extended by $20 \mathrm{~cm}$ on the downstream end. An additional benefit of this geometry is that it negates the possibility of magnetic reflection of the electrons during their downward path by ensuring decreasing field strength along that path. A diagram of the magnet, including field lines generated with the OPERA code, is shown in Fig. 1.

As the electrons propagate downward, they execute helical motion about the field lines. Depending on the initial condition of an electron when launched from the foil, it is possible that after one helical turn, it could reintercept the foil. To prevent this, the magnet was designed with a primary field component tilted by $220 \mathrm{mrad}$ from vertical about the transverse horizontal axis at the location of the foil. Thus, electrons are guided a sufficient distance below the foil in the first turn and do not reintercept it on the second turn.

The combination of these features in the second chicane magnet, along with other field specifications discussed more completely in [7], has resulted in a complicated field distribution that contains significant components in all three directions.

An electron catcher at the bottom of the aperture is responsible for absorbing the electrons in a controlled fashion. A complete design analysis of the catcher system including predicted efficiencies is available in [8]. As seen in the photograph in Fig. 2, the catcher contains five wedges arranged about a central point and mounted on a water-cooled copper plate. The design calls for the electrons to intercept the underside of the wedges where they have a high probability of absorption on the first impact. A catching efficiency of up to $99 \%$ was predicted for the optimized design system.

During the first four years of operation several modifications were made to the SNS injection region to address problems discovered after beam was turned on [9]. Two of these modifications are relevant to this study. First, the injected beam spot at the foil was moved $7 \mathrm{~mm}$ beam left of the original design to accommodate waste beam transport. Second, in 2009, the foil was moved $5 \mathrm{~mm}$ downstream to prevent electrons from intercepting the foil mount bracket. Subsequently, in 2010 the as-installed position of the electron catcher was measured and found

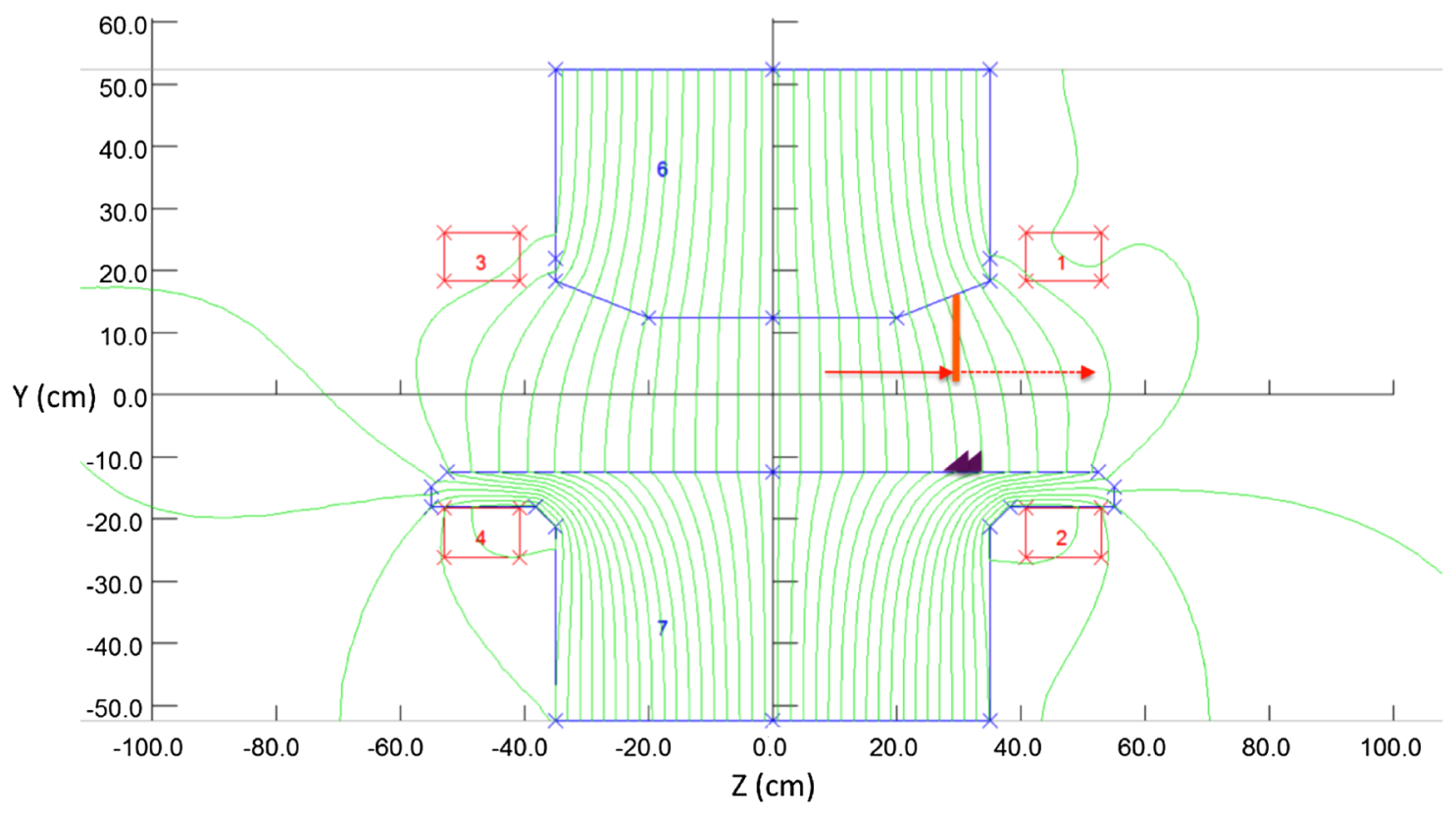

FIG. 1. The injection magnet with field lines generated by the OPERA code. Cartoon representations of the injection foil (orange line), the electron catcher (purple triangles), the incoming $\mathrm{H}^{-}$beam (solid red arrow), and the outgoing proton beam (dashed red arrow) are drawn in approximately the correct locations, but are not to scale. 

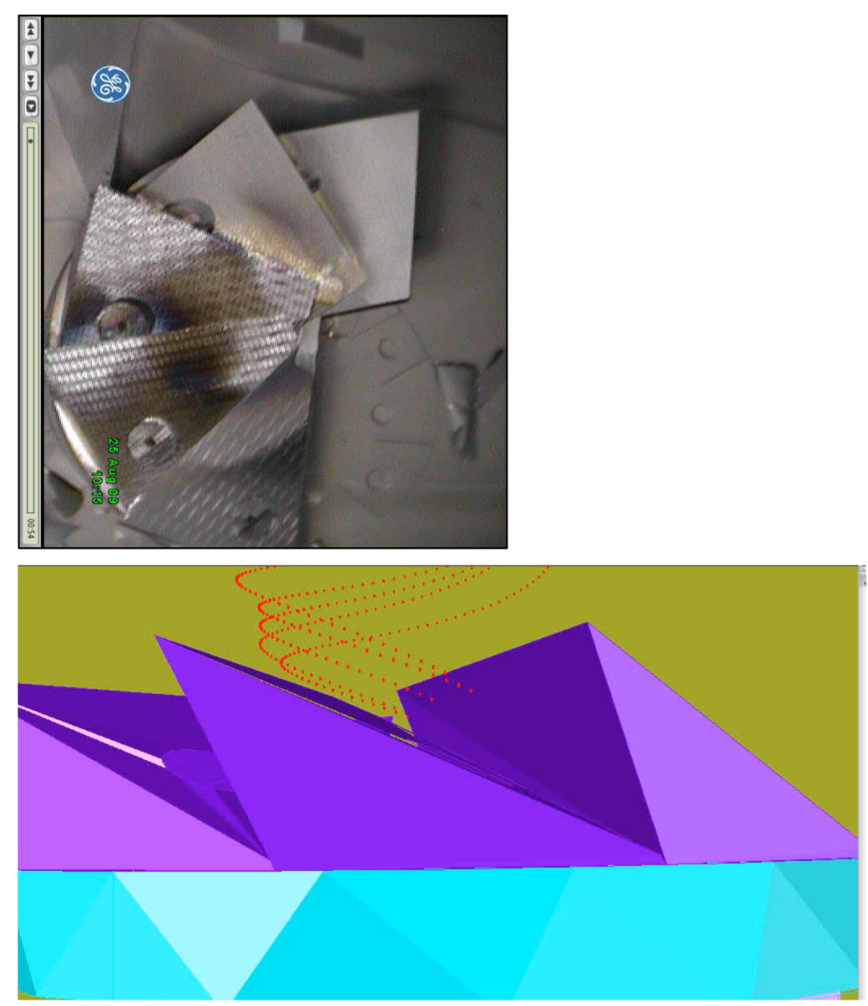

FIG. 2. (top) Photograph of the top of the electron catcher. Black marks are visible on the top surfaces where the electrons have scrubbed off the aluminum coating which resulted from foil bracket failures. (bottom) Computer-generated side view of catcher wedges, showing ideal electron trajectories as dashed red lines.

to be $14 \mathrm{~mm}$ downstream, $1 \mathrm{~mm}$ beam right, and $3 \mathrm{~mm}$ below the design specification. Accounting for the two moves of the injected beam spot position, the catcher is now located $9 \mathrm{~mm}$ downstream and $8 \mathrm{~mm}$ beam right of the design specification. By design, the position acceptance of the catcher is $\pm 2.5 \mathrm{~mm}$ in the vertical plane, $\pm 6.5 \mathrm{~mm}$ in the longitudinal plane, and a few $\mathrm{mm}$ in the horizontal plane. Thus the catcher is not, nor has it ever been, located within the design position acceptance. Owing to this, it is likely that a large fraction of the electrons intercept the top surface of the catcher wedges rather than the underside, and are subject to reflection back into the vacuum chamber.

\section{EVIDENCE OF ELECTRON CATCHER INEFFICIENCY}

Three experimental observations support the hypothesis that there is considerable catcher inefficiency in the SNS ring injection system. First, a photograph of the catcher taken in 2009 shows distinct black marks on the upper surfaces of the catcher wedges (see Fig. 2). The catcher wedges are made of carbon and were originally black in color. However, multiple foil bracket failures have deposited aluminum on the exposed areas of the catcher surfaces, changing the color and reflectivity. This has provided a fortuitous mechanism for identifying the impact locations of the electrons: Electrons impacting the surface scrub off the aluminum coating, returning the surface to the original black color. Since the catcher was designed to capture electrons on the underside of the wedges, the fact that impact spots are seen on the top surfaces signifies catcher inefficiency. Though it is impossible to estimate from this photo the fraction of electrons that intercept the top surface versus the underside, the presence of the black marks on the top surfaces indicates that it is, or it was at some point in time, a significant amount.

Second, during a vacuum entry to remove foil debris from the chamber, a photograph was taken of the top of the vacuum chamber. The photograph, shown in Fig. 3, shows a black mark on the upper chamber surface, above and slightly beam left of the foil. The mark is probably due to graphitization of the stainless steel surface by electron impacts. The photo is not clear enough to estimate the size or full extent of the mark, though there is some indication that it has a ringlike shape.

Third, a foil bracket exposed to 147 MW-hrs of integrated beam power over a 15.5 day period [10] showed

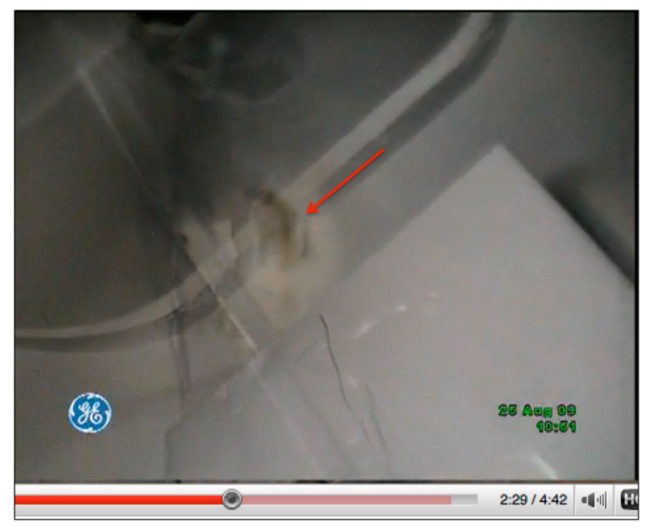

FIG. 3. Photograph of the top surface of the vacuum chamber roughly above the injection foil. The red arrow indicates the location of the edge of the black mark on the chamber surface (just left of center in image).

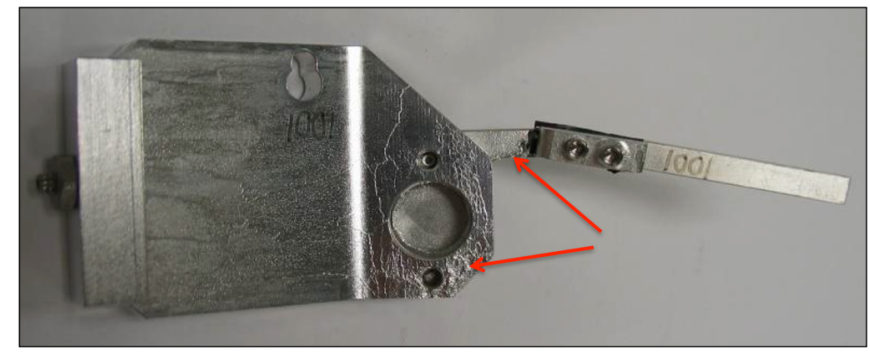

FIG. 4. Photograph of the 3rd generation foil mount assembly after it was removed from the injection chamber. The red arrows indicate the damage areas on the lower right corner of the bracket leg and the lower portion of the arm (figure reproduced from Ref. [14]). 
signs of surface damage on the bracket assembly leg and arm. The damage was severe enough to cause the bracket arm to droop. A photo of the bracket is shown in Fig. 4.

These three pieces of evidence illustrate the need for a comprehensive study of foil-stripped electron dynamics for the as-installed SNS injection system. The goal of the study is to explain the experimental observations through simulations of the stripped, uncaught electrons, and to either validate or refute the claim that the observed hardware damage is caused by these electrons. Additionally, understanding the behavior of the electron transport in the SNS $1 \mathrm{MW}$ operation is useful for the design of future high power charge exchange injection applications.

\section{ELECTRON TRAJECTORIES IN STATIC MAGNETIC FIELDS}

The intuition necessary to design a magnetic configuration capable of capturing stripped electrons lies in understanding electron motion in strong static magnetic fields. This motion can be derived using an ordering approach in which the length scale for change in the magnetic field is large compared to the electron gyroradius [11]. In this model, the electron motion is broken down into components parallel and perpendicular to the magnetic field. The motion is described in terms of the following quantities: the magnetic field $\vec{B}=B \hat{b}$, the electron mass $m_{e}$, the relativistic velocity factor $\gamma$, the velocity components parallel $v_{\text {par }}$ and perpendicular $\vec{v}_{\text {perp }}$ to the field, the parallel $W_{\mathrm{par}}=\left(\gamma m_{e} v_{\mathrm{par}}^{2}\right) / 2$, perpendicular $W_{\text {parp }}=$ $\left(\gamma m_{e} v_{\text {perp }}^{2}\right) / 2$, and total $W=W_{\text {par }}+W_{\text {perp }}$ "energies," and the electron's orbital magnetic moment $\mu=\frac{W_{\text {perp }}}{B}$.

To lowest order, the field is constant in space and the electron motion is helical along the field lines with constant velocity $v_{\text {par }}$ parallel to the field and with gyroradius $r_{g}=\frac{\gamma m_{e} v_{\text {perp }}}{e B}$ and frequency $\omega_{c}=\frac{e B}{\gamma m_{e}}$ perpendicular to the field. In the next order, the field is not uniform, so that its strength changes in space and the field lines are curved. These changes are taken to be slow in the sense that the field line curvature and $\frac{B}{\nabla B}$ are much larger than $r_{g}$. In this case, there are electron drifts superposed on the helical gyromotion. One drift, the grad- $B$ drift, is caused by the changing magnetic field strength. The velocity of the grad- $B$ drift is given by $\vec{v}_{\text {grad }}=\frac{\mu}{e} \frac{\vec{B} \times \nabla B}{B^{2}}$. A second drift, due to the curvature of the field lines, is given by $\vec{v}_{\text {curv }}=$ $\left[\left(2 v_{\text {par }}^{2}\right) / v_{\text {perp }}^{2}\right] \frac{\mu}{e} \frac{\vec{B} \times \nabla B}{B^{2}}=\left[\left(2 \nu_{\text {par }}^{2}\right) / v_{\text {perp }}^{2}\right] \vec{v}_{\text {grad }}$. The final consideration is the magnetic mirror effect $W_{\text {par }}=W-$ $\frac{B}{B^{0}} W_{\text {perp }}^{0}$, where $B^{0}$ and $W_{\text {perp }}^{0}$ are the values of $B$ and $W_{\text {par }}$ at the initial location. If the electron moves into a stronger magnetic field, it is possible for $W_{\text {par }}$ to become zero, in which case the motion of the electron along the field line reverses direction. This is commonly called electron bounce, electron reflection, or "magnetic mirror" effect, which can occur with ions as well.
Although an understanding of electron motion in terms of gyrations, drifts, and reflections is useful in conceptualizing the motion of the electrons in the field, to understand the experimental observations a computational model which includes additional effects is required.

\section{ORBIT COMPUTATIONAL MODEL}

To carry out this study, the computational model should include the following capabilities: accurate tracking of electrons in the 3D magnetic field of the injection magnet, a surface interaction model, and absorbing apertures that match the operational configuration of the SNS injection system.

The ORBIT package is an open-source PIC-style code developed for simulating beam dynamics in high intensity machines [12]. The code is well suited to the needs of this study because it contains a module for tracking particles in 3D magnetic fields. The 3D field distribution of the injection chicane used in this work was generated with TOSCA [13], and has a $0.5 \mathrm{~cm}$ sampling resolution. The ORBIT 3D particle tracker steps the particle through the magnet with a user-specified step size, taken as $0.1 \mathrm{~mm}$ in this study. The step size is approximately 1 order of magnitude smaller than the upper limit for numerical convergence of the electron motion in the magnetic field. The tracker interpolates the field values at each step and applies the appropriate momentum kick. ORBIT also calculates the drift velocities and bounce criterion as diagnostics for each tracked electron.

The particles in the simulation are launched with a transverse distribution identical to the design proton beam spot on the foil. The mean electron energy is $506 \mathrm{keV}$, inherited from the $930 \mathrm{MeV} \mathrm{H}^{-}$beam currently in operation, and the energy distribution is assumed to be Gaussian in form with sigma equal to $0.05 \%$ of the mean energy, also akin to the proton beam distribution. The mean electron energy loss in the foil is estimated to be on the order of $\sim 0.01 \%$ and is therefore neglected in this study. The parameters for the initial electron distribution are summarized in Table I.

The foil assembly in the SNS ring contains three main components: The bracket leg, the bracket arm, and the foil

TABLE I. Parameters of the injected beam distribution.

\begin{tabular}{ll}
\hline \hline Parameter & \multicolumn{1}{c}{ Value } \\
\hline Horizontal emittance & $0.221(\pi \mathrm{mm} \mathrm{mrad})$ \\
Vertical emittance & $0.221(\pi \mathrm{mm} \mathrm{mrad})$ \\
$X$ beta at foil & $10.2(\mathrm{~m})$ \\
$Y$ beta at foil & $10.8(\mathrm{~m})$ \\
$X$ alpha at foil & $0.063(\mathrm{mrad})$ \\
$Y$ alpha at foil & $0.064(\mathrm{mrad})$ \\
Mean kinetic energy & $506(\mathrm{keV})$ \\
Sigma of energy spread & $0.25(\mathrm{keV})$ \\
\hline \hline
\end{tabular}


itself. The entire assembly is tilted 30 degrees from the transverse plane about the vertical axis.

As seen in Fig. 2, the geometry of the individual catcher wedges is complex. To simplify the computational implementation, the model approximates the catcher as a single flat surface. The implications of this approximation are considered in Sec. VID. The catcher wedges are $14 \mathrm{~mm}$ tall and the top point has been measured to be $70 \mathrm{~mm}$ below the vertical magnet midplane. Therefore in the simulations the catcher surface height is varied in the range of 70 to $84 \mathrm{~mm}$ below the vertical midplane.

The 3D tracker alone is sufficient to track the electrons to the catcher and determine the resulting impact distribution. However, to calculate the capture efficiency and to prepare an electron distribution for subsequent tracking, a surface interaction model is necessary. The surface interaction model developed in ORBIT for this project is a Monte Carlo method that utilizes scattering probability distributions generated by the MCNPX code. The MCNPX model was based on $560 \mathrm{keV}$ electrons (from a $1 \mathrm{GeV}$ parent proton beam) impinging on a carbon surface with incident angles ranging from 0 to 90 degrees, in steps of 10 degrees. Integrated probability distributions for the inplane scattering (e.g., the scattering angle in the plane of incidence), the out-of-plane scattering angle, and the outgoing kinetic energy were generated. These were incorporated in ORBIT assuming no correlation among the three distributions; the impact of this assumption is discussed in Sec. VID.

Figure 5 shows the integrated probability distributions for the in-plane scattering. The angle is referenced from the normal to the plane, such that $0^{\circ}$ represents an incoming particle normal to the surface and $90^{\circ}$ represents an incoming particle parallel to the surface. Note that as the incident angle increases (shallower incidence), the in-plane scattering angle approaches mirror reflection. For the

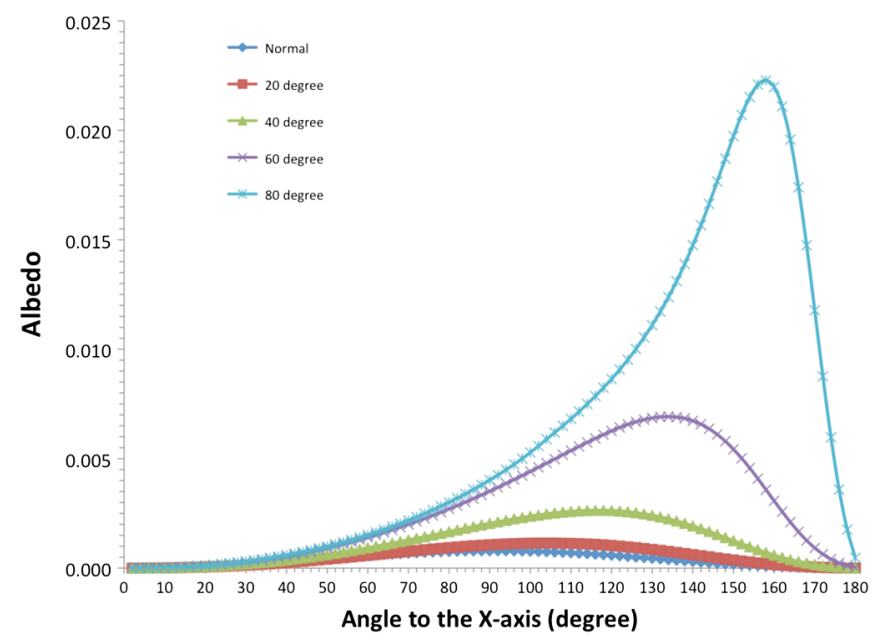

FIG. 5. In-plane scattering angle probability distributions for various incident angles (see legend on plot). Zero degrees represents the normal to the surface. problem at hand, the incident particle angle is approximately $75^{\circ}$. The surface interaction model built into ORBIT interpolates the value of the probability distribution from the bounding curves, in this case the $60^{\circ}$ and $80^{\circ}$ curves. The integrals of the probability distributions in Fig. 5 are less than one because a particle that impacts the surface may be absorbed or reflected. For example, for the probability curve nearest to the SNS electron incident angle (the $80^{\circ}$ curve), the total albedo is 0.57 , meaning that an electron with this incident angle has a $43 \%$ chance of absorption, and a $57 \%$ chance of reflection. For $60^{\circ}$ incidence, the albedo has dropped to 0.25 , and the electron is much more likely to be absorbed than reflected.

If the Monte Carlo method determines that an electron is reflected, the probability distributions for the in-plane scattering angle, the out-of-plane scattering angle, and the outgoing kinetic energy are interpolated and sampled to determine the scattered particle state. Figures 6 and 7 show the MCNPX probability distributions for the out-of-plane scattering and the outgoing kinetic energy. From these distributions we can conclude that most of the scattering is kept within the incident plane, and that the scattering is predominantly elastic in nature.

The surface interaction model was applied only to the bottom surface of the beam pipe, and only one surface interaction event was allowed per particle. The requirement of absorption on a subsequent impact is due to the fact that the surface scattering model only applies to $\sim 560 \mathrm{keV}$ electrons impinging on carbon, which is only valid for the first impact. In every subsequent case where an electron intercepts a surface or piece of injection hardware, it will be absorbed; this approximation should be sufficient to represent the gross behavior of the system. A detailed geometric representation of the relevant apertures is included in the simulation. Figure 8 gives a cartoon representation of the simulation scheme.

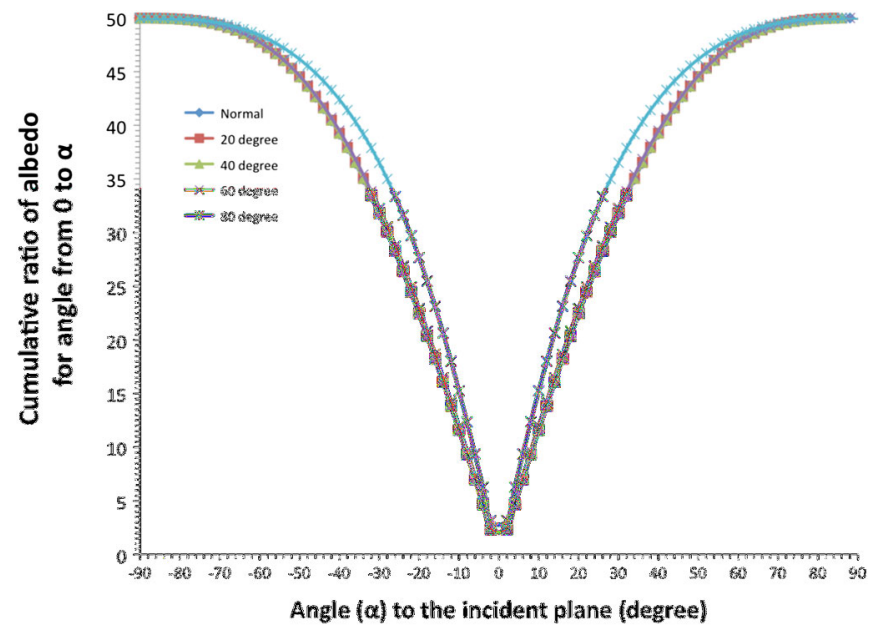

FIG. 6. Integrated probability density for the out-of-plane scattering angle for various incident beam angles. Zero degrees represents the incident plane. 


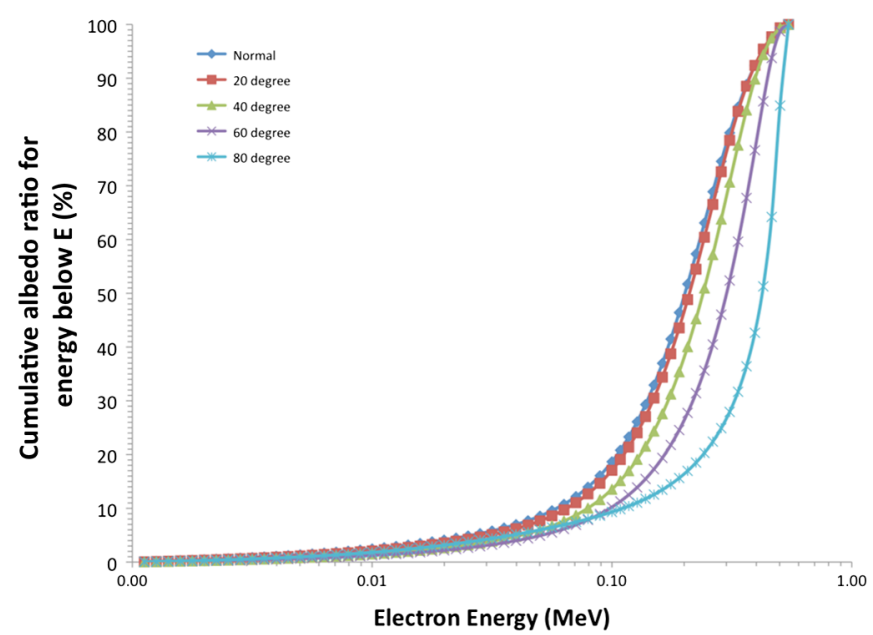

FIG. 7. Integrated probability distribution for outgoing beam energy for various incident beam angles and incident energy $560 \mathrm{keV}$.

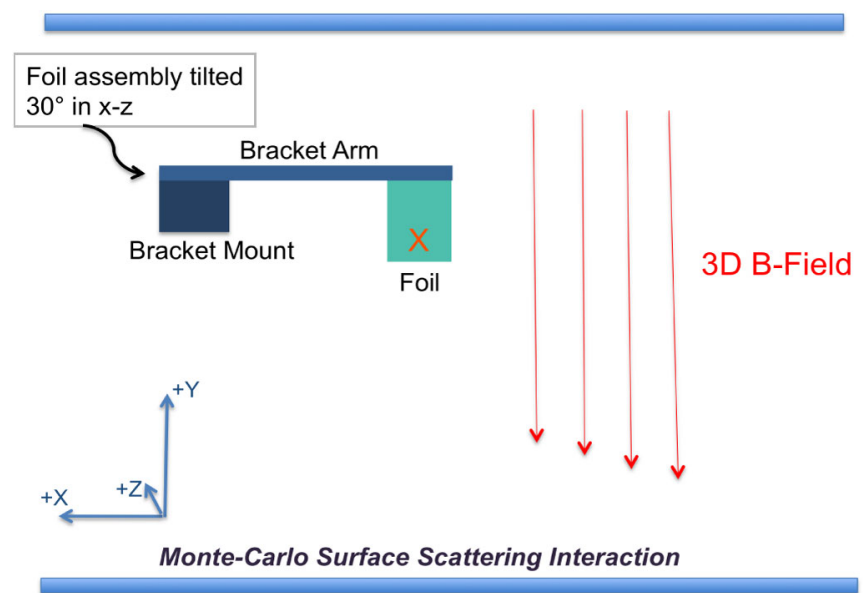

FIG. 8. Cartoon representation of the simulation configuration showing the foil assembly, the apertures, the $B$ field primary component $\left(B_{y}\right)$, and the coordinate system. Note that surface scattering occurs only on an electron's first interaction with the $-y$ aperture.

Collective effects such as the electron self-fields and the beam space charge were neglected in this work because the strength of these effects is weak compared to the strength of the external field. For instance, if one assumes a uniform density, full intensity electron beam with parameters such as those given in Table I, and allowing for a reasonable level of debunching due to the $170 \mathrm{~m}$ transport line from the linac to the ring, then the force of the electron beam on a test particle near the edge of the beam is easily calculated. The result is 4 orders of magnitude less than the force of the external field, and therefore can be neglected. Similarly, the electrons are not significantly affected by the proton beam, primarily because of their relatively high kinetic energy. LABVIEW simulations of $\sim 550 \mathrm{keV}$ electrons passing through the edge of the nominal SNS proton beam distribution indicate a deflection on the order of hundreds of microns, which is insignificant compared to the gyromotion.

Lastly, electron cloud generation was not included in this project, mainly because it was considered to be outside the scope of the study.

\section{RESULTS AND COMPARISON WITH EXPERIMENTAL OBSERVATIONS}

\section{A. Main features of electron motion}

The second injection chicane dipole magnet was designed so that the field lines at the stripper foil tilt away and down toward the bottom of the magnet where the electron catcher is located. Based on the discussion of Sec. IV, three main features characterize the initial stripped electron motion in the field of the injection chicane. First, because the field line tilt at the foil is $220 \mathrm{mrad}$ from vertical ( $y$ ), the stripped electrons execute helical gyromotion along the field lines with $\frac{v_{\text {par }}}{v_{\text {perp }}}=\tan (0.220) \approx 0.223$. The resulting electron gyroradius is approximately $12 \mathrm{~mm}$. Because of their parallel velocity, the electrons follow the $B$ field lines downward and in the positive beam longitudinal $(z)$ direction. Second, the electron motion has a positive horizontal $(x)$ component in the beam transverse plane due primarily to the gradient drift discussed above. Quantitatively, for every $1.0 \mathrm{~cm}$ of electron motion in the $-y$ direction, the gradient drift results in $0.036 \mathrm{~cm}$ of drift in the $+x$ direction. Because of the ratio $\frac{v_{\text {par }}}{v_{\text {perp }}} \approx 0.223$, the curvature drift velocity is only about $4 \%$ as large as the gradient drift, and is therefore negligible. These basic parameters of electron motion are in agreement with those of Ref. [8].

Third, the $B$ field in the magnet was specifically designed to decrease along the field lines from the stripper foil to the electron catcher, thus preventing magnetic reflection of the stripped electrons. However, for electrons which travel upward from the bottom surface after a reflection, the possibility of magnetic reflection exists, but with low probability.

\section{B. Results of ORBIT simulation for generation 4 foil assembly}

The full computational model was applied for the case of the current SNS injection configuration. The foil assembly at this time is in its 4th design generation. A photo of the assembly is shown in Fig. 9.

A 10000 macroparticle distribution generated according to the parameters in Table I was used to simulate the stripped electron beam. Figure 10 shows three different views of typical simulated electron paths from the foil down to the bottom surface, taken here as the vertical midpoint of the catcher wedge $(77 \mathrm{~mm})$. The views demonstrate the basic features of motion discussed earlier. 


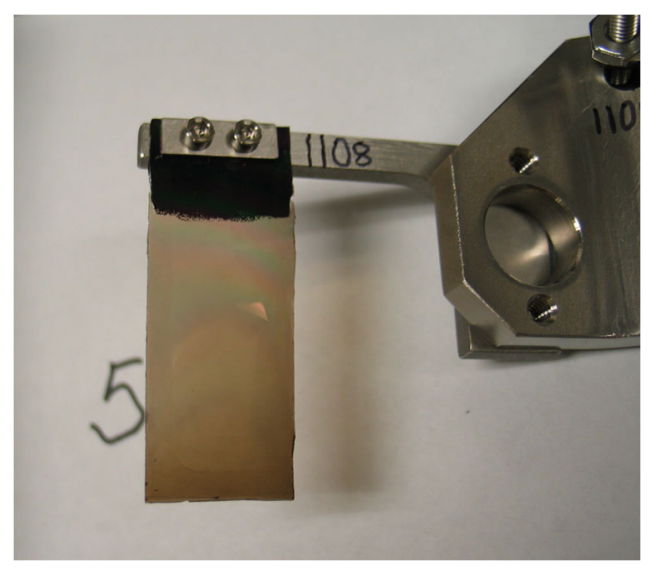

FIG. 9. Photo of the SNS 4th generation foil assembly.

Upon impact on the bottom surface, the electrons are absorbed or reflected according the surface albedo, described in the previous section. In total, there are four possible fates for the electrons: (i) absorption at the bottom surface; (ii) reflection at the bottom surface followed by absorption at the top surface; (iii) reflection at the bottom surface followed by absorption on the foil or bracket assembly; and (iv) reflection at the bottom surface followed by magnetic reflection and final absorption on the bottom surface.

Figure 11 shows the $X-Y$ view of the final impact distribution, along with example trajectories for each of the cases listed above. Note that there is a unique trajectory for each of the 10000 macroparticles, and Fig. 11 gives only a representative sample. The grad- $B$ drift motion of the gyrating electrons in the $+x$ direction can be clearly seen in both Figs. 10 and 11.

To assess the consequence of uncaught electrons on the local machine hardware, it is useful to analyze the impact distribution of these electrons. The $X-Z$ bird's eye view of the distribution, shown in Fig. 12, is perhaps the most insightful. Since the $y$ axis is not visible in this view, to interpret the plot one should recall that the electrons travel in the $+z$ direction as they pass from the foil to the bottom aperture. The foil is located at approximately $z=0.3 \mathrm{~m}$, and therefore impact spots with $z$ greater than this value are on the bottom aperture, and spots with $z$ less than this value are on the top aperture. On inspection, we see that the bottom aperture impact spot (centered at $x=0.06 \mathrm{~m}, z=$ $0.33 \mathrm{~m}$ ), which represents electrons striking the catcher surface, is very localized. This is because the energy spread in the initial stripped electron beam is small (see Table I), and all electrons travel on similar paths and intercept the surface in close proximity to one another. In contrast, during the surface reflection process the electron energy and angle are altered, resulting in a spread in the trajectories and impact locations for reflected electrons. The upper vacuum chamber impact spot is dense with a ringlike extension. The dense spot contains the peak of the proba-
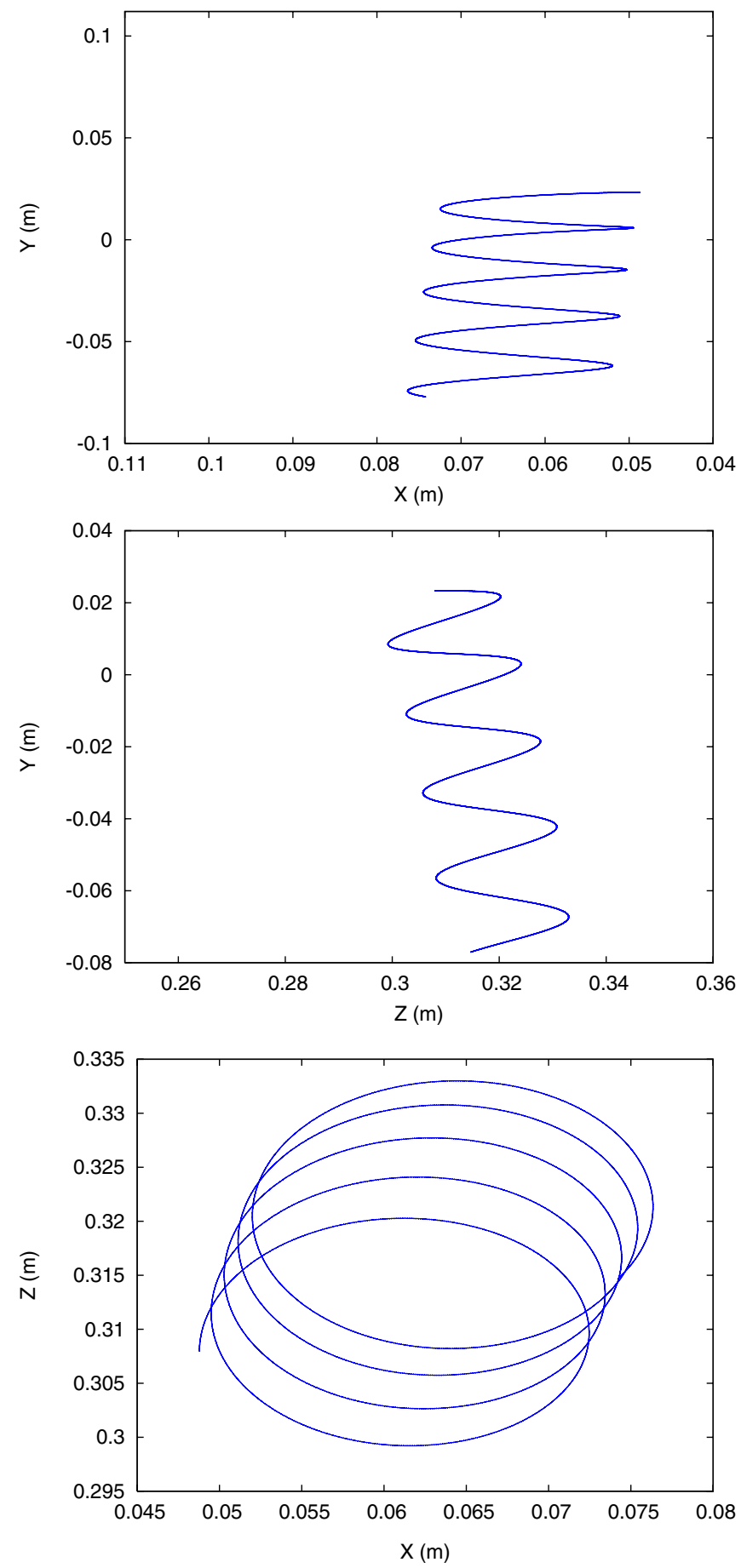

FIG. 10. Example of a typical stripped electron trajectory from the launch coordinate at the foil $(x=0.05, y=0.025, z=0.3)$ to the bottom aperture. Three views are shown: $X-Y$ (top), $Z-Y$ (middle), and $X-Z$ (bottom).

bility distributions for angle and energy, and the ring extension comes from the tails of those distributions.

In addition to the dense spot on the bottom aperture resulting from the first impact of the electrons, there is a sparsely populated ring-shaped spot located $+x$ of the main spot. This comes from electrons that were reflected 

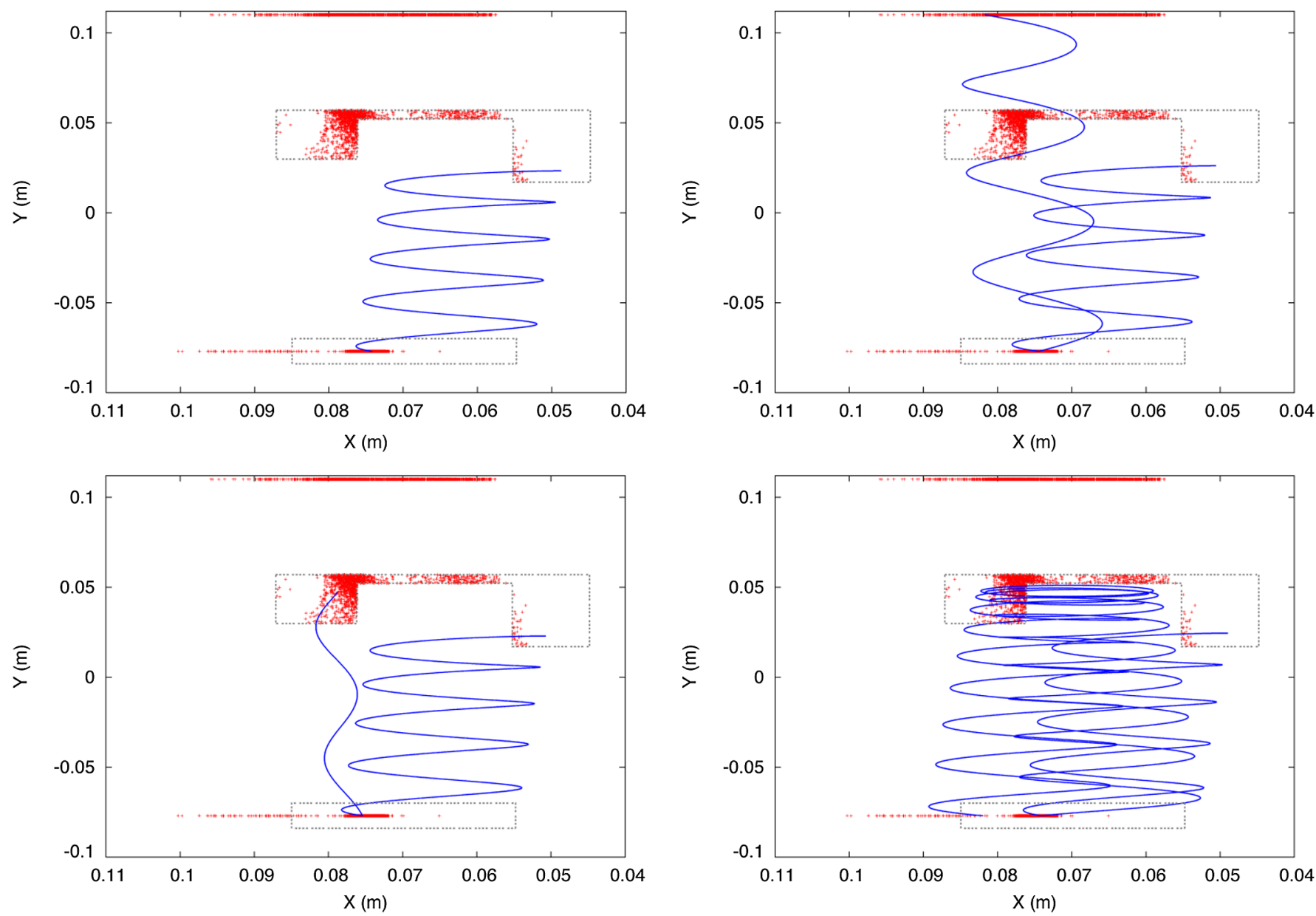

FIG. 11. $X-Y$ view of the impact distribution with four individual example electron trajectories: (top left) An electron that is absorbed on the first impact of the bottom surface, (top right) an electron that is reflected from the bottom surface and absorbed at the top surface, (bottom left) an electron that is reflected at the bottom surface and absorbed on the foil assembly, and (bottom right) an electron that is reflected from the bottom surface and then magnetically reflected by the field and finally absorbed at the bottom surface. The gray dashed line represents the foil assembly and electron catcher regions.

from the bottom surface and then magnetically reflected back downward. Finally, a significant fraction of the reflected electrons also hit the foil, bracket arm, and bracket leg, shown as the diagonal line in the center of Fig. 12.

Because of the transverse gyromotion of the electrons, the final impact distribution is sensitive to the height of the catcher, which varies from 70 to $84 \mathrm{~mm}$ below the vertical midplane of the magnet. For completeness, the simulations were run for six bottom aperture locations spanning the catcher height range. Table II summarizes the impact distribution statistics for all cases. Note that there is up to a $6 \%$ range in the impact populations due to the height variation of the bottom surface.

One of the main goals of this project is to validate or disprove the hypothesis that the experimental observations presented in Sec. III are caused by uncaught electrons. The impact distribution in Fig. 12 provides a straightforward means for comparing simulation with experimental observation. First, the photo of the electron catcher in Fig. 1 indicates distinct black marks on the top surfaces of the catcher indicating a high density of electron impacts. Though the simulation produces only one distinct high density impact location, it is important to recall that the injection spot position has undergone two major moves since the start of beam operation in the ring, as well as a number of minor moves. Therefore, black marks at several locations are anticipated. As a check, simulations were performed for the three different injection spot locations described in Sec. II. The resulting impact distribution on the catcher surface is shown in Fig. 13. An unfortunate consequence of the flat surface approximation is that it cannot accurately reproduce the relative impact locations observed in the actual system. This is because the actual catcher surface is tilted 25 degrees, such that a transverse position change corresponds to a change in the height of the catcher surface. Because of the height difference, the electrons intercept the surface at a different point in the gyromotion, leading to up to a $24 \mathrm{~mm}$ difference in transverse impact location for the tilted surface compared with a 


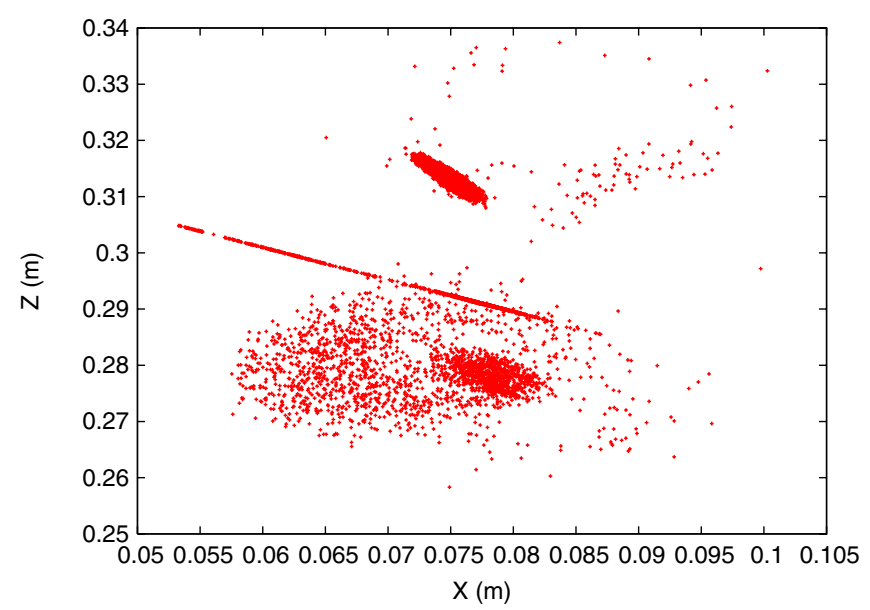

FIG. 12. $X-Z$ view of the impact distribution of 10000 stripped, uncaught electrons. The dense spot centered at $(x=$ $0.06, z=0.33$ ) shows the electrons absorbed at the first interception by the bottom surface. The sparsely populated ring to the right of this comes from electrons that are reflected from the bottom surface and then by the magnetic field, and finally absorbed on the bottom surface. The diagonal line centered at ( $x=0.06$ and $z=0.3$ ) shows electrons impacting the foil assembly. Finally, the dense spot located at $(x=0.065, z=0.29)$, with a ringlike extension shows the electrons that were reflected from the bottom surface and absorbed at the top surface.

flat surface for the same injection position. Nonetheless, the main point of interest is not the absolute positions of the impact spots but rather that they exist in multiplicity, which is observed in both simulation and experiment.

Second, the simulated impact spot on the top of the aperture in Fig. 12 can be correlated with the photo of the top of the vacuum pipe shown in Fig. 3. Unfortunately, the location of the black mark was not recorded when the photograph was taken. However, the location can be approximated and appears to be within $1 \mathrm{~cm}$ of the spot location seen in the simulation. Because of the poor lighting in the photograph, as well as the history of injection spot moves, it is not possible to resolve the shape and extent of the mark well enough to compare the parameters with the simulated impact spot. It is only possible to remark on its presence in both simulation and experiment.

The third and last piece of evidence, the damaged foil bracket, is considered in the next section.

TABLE II. Loss distribution of electrons for 4th generation foil assembly.

\begin{tabular}{lc}
\hline \hline Location & $\%$ of electrons lost \\
\hline Bottom aperture & $59-62$ \\
Top aperture & $23-28$ \\
Foil & $1-5$ \\
Foil assembly bracket arm & $3-4$ \\
Foil assembly bracket leg & $4-10$ \\
\hline \hline
\end{tabular}

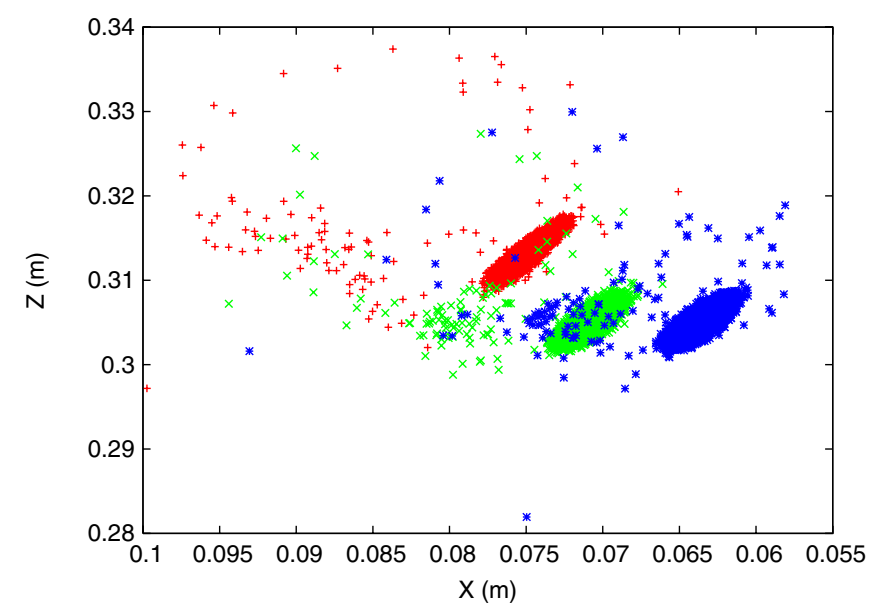

FIG. 13. $X-Z$ view of the bottom aperture impact distribution for the three different injection spot positions described in Sec. II. The blue dots correspond to the design injection position, the green dots correspond to a move of the injection spot $7 \mathrm{~mm}$ beam left, and the red dots correspond to the final move of the injection position $5 \mathrm{~mm}$ downstream.

\section{Results of high statistics simulation for generation 3 foil assembly}

The 3rd generation foil assembly configuration was simulated because damage was observed on the bracket leg and arm after exposure to $147 \mathrm{MW}$-hrs of beam over a two week period (see Fig. 3). In fact, this damage prompted design changes to the assembly. Specifically, the main differences between the 3rd and 4th generation foil assembly are the position of the foil on the bracket arm, which was increased by $1 \mathrm{~cm}$ in the 4th generation, and the material, which was switched from $\mathrm{Al}$ to Ti. The material properties of $\mathrm{Ti}$, including the coefficient of thermal expansion and melting point, are better matched to the situation [14]. A photograph of the 3rd generation assembly taken prior to exposure to beam is shown in Fig. 14.

The simulation results for the 3rd generation indicate substantially more losses on the foil assembly than the 4th generation assembly, particularly on the bracket leg. This is due to the shorter arm length which places the bracket

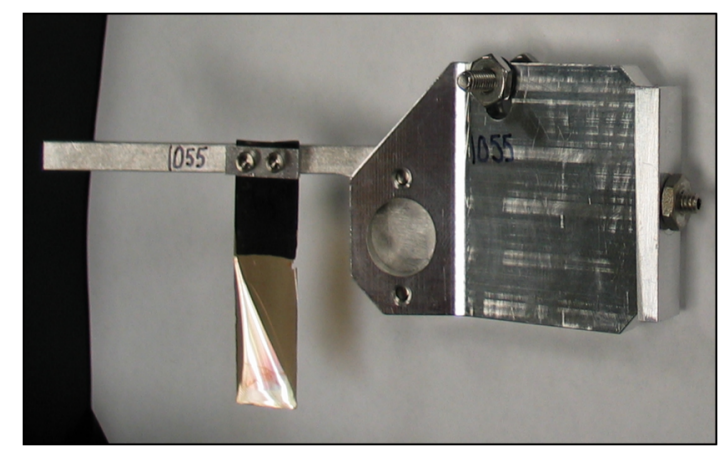

FIG. 14. The 3rd generation foil assembly before use with beam. 
TABLE III. Loss distribution of electrons for 3rd generation foil assembly.

\begin{tabular}{lc}
\hline \hline Location & $\%$ of electrons lost \\
\hline Bottom aperture & $58-63$ \\
Top aperture & $7-26$ \\
Foil & $3-17$ \\
Foil assembly bracket arm & $0-1$ \\
Foil assembly bracket leg & $7-25$ \\
\hline \hline
\end{tabular}

leg in a higher probability region of scattered electrons. The full statistics of the impact distribution are given in Table III, again for the entire range of catcher heights. The $x-y$ view of the impact distribution for the midplane catcher height is shown in Fig. 15. The impact location of the electrons on the bracket coincides with the damaged regions (see Fig. 4), thus supporting the notion that this damage was caused by scattered electrons. The extension of the arm by $1 \mathrm{~cm}$ in the 4th generation assembly reduces the number of electrons hits on the assembly (see for instance Table II), but does not entirely eliminate the problem. However, the assembly is less sensitive to damage from the remaining hits because of the new Ti material.

\section{Error estimates}

As described in Sec. V, in order to simplify the computational implementation, the model contains a number of approximations to the actual system. Most of these approximations are of negligible consequence, with the exception of one or two. In these cases, it is prudent to estimate the errors associated with these approximations.

The most significant approximation is the flat surface representation of the catcher wedge. In reality the catcher wedges are tilted up 25 degrees with respect to the normal of a flat surface. The electron angle of incidence with the surface will be different for the tilted wedge than for the flat surface. Recalling from Fig. 5 that the distribution of scattering angles is dependent on the initial angle of incidence, it is clear that an error on the incoming angle will affect the accuracy of the scattering model. For the in-plane scattering, we see that the scattering distribution is peaked near mirror reflection, such that a shallower incident angle results in a shallower outgoing angle, etc. For the out-ofplane scattering, the distribution is strongly peaked near zero, but in the case of the tilted wedge surface, the zero is defined by the normal to the surface of the $25^{\circ}$ wedge. The net effect is that the out-scattered particle angles are different for the tilted wedge than the flat surface, resulting in an error on the initial launch condition of the scattered particle.

To mimic the effect of the tilt, the in-plane and out-ofplane scattering distributions were offset by a constant degree value. By design, the tilt of the catcher wedge closely matches the pitch of the electron trajectory (see, for example, Fig. 2), so the most likely scenario is that the

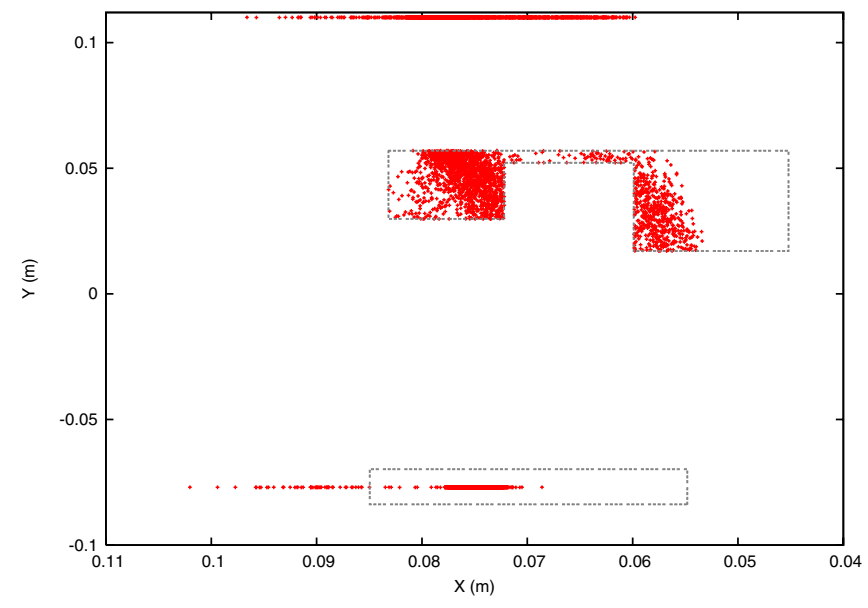

FIG. 15. Impact distribution for the 3rd generation foil assembly. The gray dashed line represents the foil assembly and electron catcher regions.

electron intercepts the catcher wedge at near grazing angle. Thus, the in-plane distribution was shifted by -15 degrees which results in a grazing angle to the surface. The unlikely case of a "head on" collision with the wedge surface was considered for completeness by shifting the in-plane distribution 25 degrees. The out-of-plane distribution was shift by both 25 and -25 degrees. To bracket the behavior, all combinations of the aforementioned in-plane and out-of-plane shifts were simulated. Table IV presents the resulting impact distributions, given as absolute increases or decreases relative to the nominal case presented in Table II.

As seen, for most cases the impact of the mimicked tilt effect is relatively small, with variations of less than a few percent from the nominal flat surface case. This result is not surprising since the centroid of electron motion is governed by the magnetic field lines, and only the radius of the gyromotion and the pitch of the helix are affected by the launch condition. A single notable consequence is that electrons outscattered at shallower angles relative to field lines are more likely to suffer from magnetic reflection, resulting in a larger population of electrons returning to the bottom surface. For the likely scenario of particles intercepting the surface near grazing angle due to the tilted catcher surface, this effect results in a 7\%-8\% increase in the population of electrons lost at the bottom surface, and a corresponding decrease of those lost at the top surface. There is no significant correlation with the offset the outof-plane distribution.

A related consequence of the flat surface approximation is the error in the surface albedo, i.e., the probability of electron absorption on the surface. For the likely case where incident angle is shallower due to the tilted wedge surface, there will be an increase in particle reflection and a decrease in absorption. This may be partially offset by the fact that the reflected particles have a higher probability of magnetic reflection, as discussed previously. 
TABLE IV. Loss distribution of electrons for 4th generation foil assembly with shifted outscattering distributions. The numbers are given as absolute increases or decreases from the results in Table II.

\begin{tabular}{lcccccc}
\hline \hline In-plane shift (deg) & Out-of-plane shift (deg) & $\%$ bottom & $\%$ top & $\%$ bracket & $\%$ arm & $\%$ foil \\
\hline-15 (grazing) & -25 & +8 & -7 & 0 & -2 & +1 \\
-15 (grazing) & 25 & +7 & -6 & -1 & 0 & 0 \\
25 & -25 & -2 & +3 & +1 & -2 & 0 \\
25 & 25 & -2 & +2 & +2 & -2 & 0 \\
\hline \hline
\end{tabular}

Finally, one other model approximation that can lead to errors is the assumption of no correlation between the inplane scattering angle, the out-of-plane scattering angle, and the outgoing electron energy. In reality, there is a strong correlation between the out-of-plane scattering angle and the outgoing electron energy. Specifically, inelastic interactions in the surface result in both energy loss and out-of-plane scattering, such that the low energy tail of the energy distribution is correlated with the tails of the out-of-plane scattering distribution. However, because the out-of-plane angle does not contribute appreciably to the electron dynamics, it is acceptable to neglect this correlation.

\section{DISCUSSION}

The results of simulations clearly support the hypothesis that stripped, uncaught electrons are, or have at one point, impacted hardware in the SNS injection region. As noted in [8], the efficiency of the catcher is sensitive to its location with respect to the injected beam position. Given the observations of black marks on the catcher surface, and the fact that the catcher has never been located within the design position acceptance, it can be reasoned that most of the electrons are impacting the top surface of the catcher. This has significant consequences in terms of beam power deposition on the local hardware. As an example, for the current SNS injection system, if one assumes that all the electrons constitute catcher inefficiency (e.g., none are properly caught by the catcher), then according to Table II, approximately $2.5 \%$ of all foil-stripped electrons will be lost on the bracket leg, and $4.7 \%$ will be lost on the bracket arm. Neglecting energy loss during the surface scattering process and scaling from the initial electron beam power of $\sim 1.07 \mathrm{~kW}$, this translates to $27 \mathrm{~W}$ of power being deposited on the bracket leg, and $50 \mathrm{~W}$ on the bracket arm. Reference [14] indicates that just $10 \mathrm{~W}$ of beam power on the corner of the $\mathrm{Al} 3 \mathrm{rd}$ generation bracket leg is enough to cause melting damage. The Ti used in the 4th generation bracket assembly is more resilient to this problem, and so far no damage has been observed. Nonetheless, the electron beam power deposition is significant and efforts are being made to reduce this through the fabrication of a new electron catcher.

Extrapolating to future beam powers in the tens of MW, the power deposition corresponding to the stripped electrons becomes even more appreciable. Care will have to be taken to either ensure that the catching is done with very high efficiency, or that the hardware in the injection region is capable of handling the load, or both. As the SNS experience indicates, it is very difficult to predict during the design phase the exact parameters for the beam injection; unforeseen complications often arise and accommodations must be made. The ideal catcher system for future machines should be robust against such changes.

At SNS, a new catcher system is being designed and will be installed to match the operational injection spot location, which has finally settled. The catcher will be robust against first pass miss, and will have a larger error tolerance to the beam spot position. The work presented in this document has resulted in a benchmarked computational tool capable of simulating the stripped electron trajectories. To aid in the design of the new catcher system, electron trajectories were generated for an ellipse of injection spot positions centered about the current injection position. The trajectories were imported into the catcher design software to visually inspect the impact location of the electrons on the catcher. Furthermore, the simulations are being utilized in the design effort of the new injection chicane magnet for the beam power upgrade project [15]. The main thrust of this work is to verify that the new magnetic fields do not cause unacceptable electron behavior, such as electron bounce on the path to the catcher, or electrons intercepting the foil after one turn of gyromotion. To conclude, the simulations have become an important tool in the design of future SNS injection region systems.

\section{ACKNOWLEDGMENTS}

The authors would like to thank T. Hunter and J. Error for their help in generating figures, and W. Blokland for his electron profile monitor simulation. ORNL is managed by UT-Battelle, LLC, under Contract No. DE-AC0500OR22725 for the U.S. Department of Energy.

[1] G. Budker et al., At. Energ. 19, 507 (1965).

[2] T. Gorlov et al., Phys. Rev. ST Accel. Beams 13, 074002 (2010).

[3] V. Danilov et al., Phys. Rev. ST Accel. Beams 10, 053501 (2007). 
[4] Y. Takeda et al., in Proceedings of the IPAC'10 Conference, Kyoto, Japan (ICR, Kyoto, 2010), p. 202.

[5] R.W. Shaw et al., in Proceedings of the IPAC'10 Conference, Kyoto, Japan (Ref. [4]), p. 3563.

[6] The MCNPX Team, MCNPX User's Manual version 2.5.0, Los Alamos National Laboratory Report No. LA-CP-050369, 2005.

[7] D. T. Abell et al., in Proceedings of the European Particle Accelerator Conference, Vienna, 2000 (EPS, Geneva, 2000), p. 2107.

[8] L. Wang et al., Phys. Rev. ST Accel. Beams 8, 094201 (2005).

[9] M. Plum, Proceedings of the ICFA HB2008 Workshop, Nashville, p. 268.
[10] M. Plum, in Proceedings of the IPAC'10 Conference, Kyoto, Japan (Ref. [4]), p. 3969.

[11] K. Miyamoto, Plasma Physics and Controlled Nuclear Fusion (Springer-Verlag, Berlin, 2005); N. A. Krall and A. W. Trivelpiece, Principles of Plasma Physics (McGraw-Hill, New York, 1973).

[12] J. A. Holmes, in Proceedings of the IPAC'10 Conference, Kyoto, Japan (Ref. [4]), p. 1901.

[13] J. G. Wang, Phys. Rev. ST Accel. Beams 9, 012401 (2006).

[14] M. Plum et al., Phys. Rev. ST Accel. Beams 14, 030102 (2011).

[15] S. Henderson, in Proceedings of the 10th European Particle Accelerator Conference, Edinburgh, Scotland, 2006 (EPS-AG, Edinburgh, Scotland, 2006), p. 345. 